\title{
Extraordinary Clouds
}

\section{Skies of the unexpected from the beautiful to the bizarre}

\author{
Richard Hamblyn
}

\begin{abstract}
A près le succès mérité de son livre L'invention des nuages. Comment un météorologue amateur a découvert le langage du ciel ( $L$ attès, 2003), Richard Hamblyn semble s' être découvert une passion pour les nuages. Il nous a donné successivement The Cloud Book. How to understand the skies (voir la critique de sa traduction française Nuages - le guide d'identification, dans le ${ }^{\circ} 67$ de la revue, p. 58), cet Extraordinary Clouds, et il va publier en mai 2010 The Met O ffice Pocket Cloud Book, chez le même éditeur.
\end{abstract}

Extraordinary Clouds est un peu surprenant, car l'auteur y présente des nuages qui sont assez extraordinaires en ce sens que la probabilité que nous puissions voir certains d'entre eux est plutôt faible, outre évidemment leur aspect étonnant ou leur beauté. Je pense en particulier à la dernière photographie, celle d'un nuage de bang sonique ; on y voit un avion de chasse dont l'arrière est caché dans un nuage en forme d'œuf et dont I' habitacle est coiffé d'une huppe nuageuse, le tout produit par le passage du mur du son. La photographie n'est pas ce que I'on appelle une belle photo, mais elle est singulière, c'est la première fois que je vois un tel nuage.

Les photographies présentées ne sont pas toutes des œuvres d'art, souvent (mais pas toujours) parce qu'elles ont été faites dans des conditions difficiles ou par des amateurs. M ais elles sont aussi très intéressantes, voire uniques en leur genre.

Le livre est organisé en cing chapitres, les nuages vus d'en haut, les formes étranges, les effets optiques, les ciels théâtraux et les nuages anthropiques. II se termine par un index, les crédits des photographies et une bibliographie. Chaque chapitre est illustré de 16 à 20 photographies, il y en a 88 en tout.
Dans le chapitre « Les nuages vus d'en haut », il y a le sempiternel cirrus accompagnant le jet-stream au-dessus de l'Égypte, photo qui date de 1966, dans une reproduction de piètre qualité. Heureusement que quelques belles allées de von Karman viennent égayer l'ensemble.

Dans les formes étranges, on trouve plusieurs piles d'assiettes (en français dans le texte) des quatre coins du monde et un splendide nuage capuchon qui couvre le sommet du montA rarat.

Parmi les effets optiques, I'éditeur n'a pas été capable de trouver un arc-en-ciel double de qualité, mais il présente une intéressante gloire d'avion assez rare, des hal os assez quelconques, mais un arc circumzénithal vu au travers d'un trou (fallstreak hole) dans un nuage stratiforme.

soient pas réellement des nuages, il y a trois photos assez extraordinaires de condensations dues aux avions; celle du bang sonique déjà cité, une photo de la condensation qui se produit le long du vortex engendré par les hélices d'un quadrimoteur et celle qui se forme sur l'extrados des ailes d'un chasseur qui cambre sa trajectoire. Ces photos sont absolument extraordinaires, les amateurs d' aéronautique apprécieront.

Je retiendrai aussi de ce livre l'accent mis sur ce que les A nglo-Saxons appellent le « fallstreak hole », terme dont je ne connais pas la traduction française. II s'agit d' un trou, parfois de forme circulaire, qui se produit dans un nuage étendu, lié à la condensation locale de gouttelettes d'eau surfondue qui précipitent, engendré par divers phénomènes, dont des ondes de gravité. II reste des désaccords sur leur origine exacte. On trouve sur Internet un grand nombre d'exemples de tels nuages ; il suffit de taper « fallstreak cloud» dans votre moteur de recherche préféré pour en trouver en quantité. Je pense en particulier à ce magnifique cirrus en forme de plume vu à travers un trou circulaire que vous ne manquerez pas de découvrir sans trop d' efforts.

Chaque photographie est accompagnée d'un commentaire écrit par un spécialiste. L'ensemble est agréable, mais l'éditeur aurait pu faire un effort pour trou-

Dans « Les ciels théâtraux », on peut retenir quel ques exemples de nuages lenticulaires tout à fait inhabituels, parmi des photos de tornades, de tempêtes de sable et de phénomènes extrêmes, pas toujours très attrayantes.

Dans « Les ciels anthropiques », j'ai retenu les «fumulus » et les traînées de condensation qui dessinent des partitions musicales dans le ciel, et beaucoup d'autres figures. Bien que ce ne ver de meilleurs exemplai res de certains phénomènes. $M$ ais il est vrai qu'il a fait un effort sur le prix du livre.

\section{Michel Rochas}

Extraordinary Clouds - Skies of the unexpected from the beautiful to the bizarre Par Richard Hamblyn

David et Charles, 2009, 144 p., 7,77 € 